\title{
JET Diagnostic Enhancements in Preparation for DT Operations
}

\author{
J. Figueiredo ${ }^{1,2}$, A. Murari ${ }^{1,3}$, C. Perez Von Thun ${ }^{1,4}$, D. Marocco ${ }^{5}$, M. Tardocchi ${ }^{6}$, F. $_{\text {. }}$ \\ Belli $^{5}$, M. García Muñoz ${ }^{7}$ A. Silva ${ }^{2}$, S. Soare ${ }^{8}$, T. Craciunescu $^{9}$, M. Santala $^{10}$, P. \\ Blanchard $^{11}$, D. Croft ${ }^{12}$ and JET contributors
}

EUROfusion Consortium, JET, Culham Science Centre, Abingdon, OX14 3DB, UK

\author{
${ }^{I}$ EUROfusion Programme Management Unit, Culham Science Centre, Abingdon, United Kingdom \\ ${ }^{2}$ Instituto de Plasmas e Fusão Nuclear, Instituto Superior Técnico, Universidade de Lisboa, Portugal \\ ${ }^{3}$ Consorzio RFX (CNR, ENEA, INFN, Universita' di Padova, Acciaierie Venete SpA), Padova, Italy \\ ${ }^{4}$ Max-Planck-Institut für Plasmaphysik, D-85748 Garching, Germany \\ ${ }^{5}$ Unità Tecnica Fusione - ENEA C. R. Frascati,via E. Fermi 45, 00044 Frascati (Roma), Italy \\ ${ }^{6}$ IFP-CNR, via R. Cozzi 53, 20125 Milano, Italy \\ ${ }^{7}$ Universidad de Sevilla, Sevilla, Spain \\ ${ }^{8}$ The National Institute for Cryogenics and Isotopic Technology, Ramnicu Valcea, Romania \\ ${ }^{9}$ The National Institute for Laser, Plasma and Radiation Physics, Magurele-Bucharest, Romania \\ ${ }^{10}$ Aalto University, P.O.Box 14100, FIN-00076 Aalto, Finland \\ ${ }^{11}$ Ecole Polytechnique Fédérale de Lausanne (EPFL), CRPP, CH-1015 Lausanne, Switzerland \\ ${ }^{12}$ CCFE, Culham Science Centre, Abingdon, Oxon, OX14 3DB, UK \\ E-mail: joao.figueiredodeuro-fusion.org
}

\begin{abstract}
In order to complete the exploitation of the JET ITER-like Wall and to take full benefit from deuterium-tritium experiments on JET, a set of diagnostic system refurbishments or upgrades is in progress. These diagnostic enhancements focus mainly on neutron, gamma, fast ions, instabilities and operations support. This effort intends to provide better spatial, temporal and energy resolution while increasing measurement coverage. But also previously non existing capabilities, such as Doppler Reflectometry, will now be made available for scientific exploitation. Guaranteeing diagnostic reliability and consistency during the expected DT conditions is also a critical condition for the work and systems being implemented. An overview of status and scope of the ongoing projects is presented.
\end{abstract}

First EPs Conference on Plasma Diagnostics - $1^{\text {st }}$ ECPD

14-17 April 2015,

Villa Mondragone, Frascati (Rome) Italy

* See the Appendix of F. Romanelli et al., Proceedings of the 25th IAEA Fusion Energy Conference 2014, Saint Petersburg, Russia 


\section{Introduction}

JET next D-T campaign, DTE2, is presently scheduled for the year 2017. From a point of view of diagnostics developments, for many years JET diagnostics have been upgraded in order to provide adequate support for the scientific exploitation of a D-T campaign [1,2,3], with particular attention to the issues posed by the neutron yield and the new wall materials [4]. The main efforts have concentrated on improving three main aspects of JET measuring capability: 1) the quality of the measurements of the electron and ion fluids to support the plasma physics programme 2) the diagnostic for the fusion products 3 ) diagnostic technologies for ITER.

In terms of general diagnostic capability, compared to the previous DTE1, JET diagnostics have a much better spatial and temporal resolution of both the ion and electron fluid (about one order of magnitude improvement for each parameter). The consistency of the various independent measurements of the same parameters has also increased significantly; the three independent measurements of the electron temperature, for example, agree now within 5\%. Moreover, solutions are being addressed to operate some cameras, both visible and IR, even during the full D-T phase to provide imaging of the plasma and the first wall. Various upgrades of neutral particle analysis are being considered, mainly to measure the isotopic composition. A new set of reflectometers is expected to provide valuable information about the changes in the turbulence with the different fuel mixtures.

With regard to the fusion products, JET now can deploy a consistent set of techniques to measure the neutron yield and neutron spectra and to diagnose the fast particles. Vertical and horizontal lines of sight are foreseen for neutron and gamma spectrometry, in order to better determine the thermal neutron yield and to separate the trapped and passing components of the alphas. Various gamma ray spectrometers are being developed to cover all the various operational scenarios, from trace tritium to 50-50 D-T operation. The redistribution of the alphas will be measured with the gamma ray cameras, recently upgraded with full digital electronics; new detectors are being considered to bring the time resolution of the system in the ten of ms range. The lost alphas will also be diagnosed with improved spatial and temporal resolution, using Faraday cups and a scintillator probe. The interaction between the alpha particles and various instabilities, particularly Toroidal Alfven eigenmodes will be studied with a set of specific antennas.

\section{Diagnostics Upgrade for Operation}

The main upgrades of JET diagnostic capability for operation regard cameras, for the monitoring plasma wall interactions, and the Neutral Particle Analyser, to determine the fuels mixture.

\subsection{Imaging}

The JET visible and infrared cameras are presently installed at a very close distance to the tokamak (typically 2 - 4 meters from the plasma boundary). The neutron yield during 50-50 D-T operation is likely to cause irreversible damage to these cameras or in the best case white out the sensor during a plasma pulse. Shielding these cameras from the neutrons is the only way to keep these diagnostics functional during D-T operations. This will be achieved by relocating the cameras to a low radiation environment behind the biological shield, and by relaying the emitted light out of the torus hall (which involves path lengths of order 20-25m) through a carefully designed system of mirrors and lenses. Due to resource limitations, this will be done only for three out of the eleven views currently in use: two wide-angle views in different sectors of the machine and one divertor view from the top of the machine, carrying information in the visible, near-Infrared and mid-Infrared wavelengths. In the torus hall, the use of lenses has been minimized to maximise the transmittance of the system and avoid darkening by radiation, although a few lenses will remain. An interesting by-product of the DT campaign will be that it will provide confirmation on whether radio-luminescence of sapphire lenses in the near- and mid-IR range is a problem or can be neglected. Several sets of moderately sized $(<500 \mathrm{~mm}$ diameter) Al- or Au-mirrors with protective coating arranged in dog leg formation will be used, with the latter material providing the highest reflectivity in the infrared range, whereas the former preserves the capability to include visible light instruments with a modest penalty to the infrared measurement. 


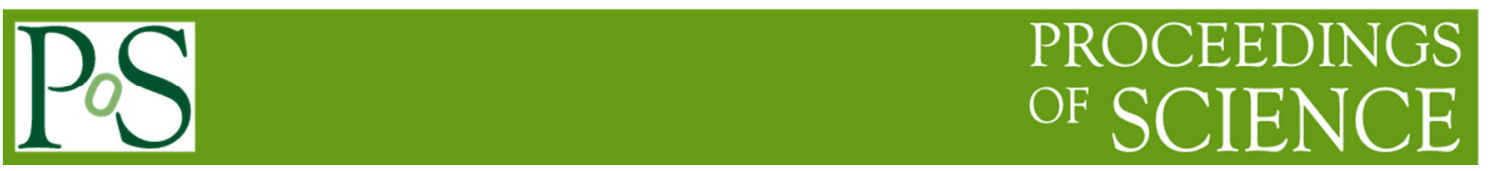

\subsection{Isotopic composition with NPA}

In the DT campaign, the main physics goal of this instrument is to analyse the isotopic composition of the plasma. Novel techniques utilizing knock-on ions could also provide useful information and it would be important for ITER to test the feasibility of such techniques. In addition to plasma composition studies, the NPA has found use in the study of slightly RF accelerated tails.

The diagnostic is being enhanced to upgrade the ion detection and data acquisition systems to obtain the best possible scientific results, addressing the most important issues affecting the performance, like count rate capability at low energy channels and sensitivity to radiation background. Presently, pile-up degrades data quality from about 10kcps.

Previously, thin CsI(Tl) scintillators coupled to photomultipliers have been used as ion detectors. Their drawbacks are relatively high sensitivity to neutron-induced X-rays and limited pulse-height resolution making signal/background discrimination difficult. On top of that, they are slow with 3 us decay time. The diagnostic will be equipped with thin custom-made Si detectors optimized for ion detection on JET NPAs. The detectors are designed as silicon-on-insulator structure with the active layer ground to only a few microns thickness but supported by a thick substrate to create a robust detector. Very similar detectors have been deployed in a second NPA instrument at JET, where they demonstrated a very weak response to JET radiation background. The detectors also allow improved adaptation to different neutral emission rates, as selective masking of strips permits changing the effective active area under software control. First tests with the recently manufactured detectors have been performed, which for relevant bias voltages showed leakage currents well below the 100nA set as tolerance limit. MCNP simulations have been performed to quantify the sensitivity to background radiation and assess the anticipated performance in DT conditions. Results from these simulations have been incorporated into the detector design, in particular for the choice of substrate material and detector thickness.

To further minimize the noise, the detector and readout chips (based on PORGAMRAYS from Rutherford-Appleton-Laboratory) will be in close proximity and integrated into in-vacuum electronics PCBs. This will be supplemented by FPGA-based communications and processing electronics on the air-side for controlling the readout chips and receiving and re-transmitting the readout data for final storage. With the new planned readout, the detectors can count up to $200 \mathrm{kcps}$, and more if strips are disabled on pulse-topulse basis. This has largest impact on evaluating the close-to-edge composition where NPA generates the most reliable data, and for hot, high density plasma with high neutral emission rates.

\section{Diagnostics upgrades for neutron detection}

\subsection{Neutron Spectrometry}

The implementation on JET of a dedicated compact neutron spectrometer started in mid-2007 and was commissioned during 2012. It was led by ENEA who built a Digital Pulse Shape Discrimination (DPSD) board, coupled to a NE213 scintillator detector (diameter $2.5 \mathrm{~cm} \times 2.5 \mathrm{~cm}$ thick) with LED for photomultiplier gain variation corrections, built by Physikalisch-Technische Bundesanstalt (PTB) [5].

A new enhancement project with the goal to install a similar spectrometer in the Roof Lab has been ongoing to address the fact that JET has not yet a $14 \mathrm{MeV}$ compact neutron spectrometer, suitable for DT plasma Campaigns, on a vertical, radial line of sight. The magnetic field in a tokamak provides a preferred direction in space, about which the charged particles in the plasma must orbit; the energy spectra of the fusion reaction neutrons vary with the spectrometer viewing angle and it is therefore possible to extract more information on these reactions using different lines of sights (e.g. horizontal and vertical). In particular, a vertical view gives the best separation of neutron spectra from RF-driven and thermal sources, due to the RF-driven ion motion being mainly in the vertical poloidal plane. This applies also to neutron spectra from synergetic NBI plus RF-driven sources. On the other hand, a horizontal view gives the best separation of neutron spectra from NBI and thermal sources, as the NBI injection is pseudo tangential in the toroidal plane and gives rise to co- or counter streaming ion populations.

Together with the NE213 scintillator detector, a diamond detector suitable for $14 \mathrm{MeV}$ neutron measurements at very high count rates $(>1 \mathrm{MHz})$, will be installed in front of the NE213 detector and along the same line of view of TOFOR (Time-Of-Flight neutron spectrometer at Optimized Rate). The installation of the two new spectrometers is compatible and does not interfere with the existing TOFOR spec- 
trometer, nor will the diamond detector, due to its low efficiency and thickness, interfere with the NE213 scintillator.

The diamond detector is a matrix of Single crystal Diamond Detectors (SDD) in order to be able to cover the largest fraction of the incoming neutron beam (about $10 \mathrm{~cm}^{2}$ ). The matrix will be made of 12 SDD and the electronics for 10 channels will be provided. Each SDD features a total efficiency to 14 $\mathrm{MeV}$ neutrons of about $1 \%$ and an energy resolution of $\sim 5 \%$. The latter is mainly limited by the fast preamplifier and is a compromise between the capability to operate at very high count rates $(>1 \mathrm{MHz})$ without pile-up events and the adequate charge collection efficiency needed to achieve the desired energy resolution.

\subsection{Neutron Tomography}

The Neutron Camera is a JET diagnostic with the main function of measuring the neutron emissivity profile due to $2.5 \mathrm{MeV}$ (DD) and $14 \mathrm{MEV}$ (DT) neutrons over a poloidal plasma cross-section using lineintegrated measurements along a number of collimated channels (lines-of-sight, LOS).

The JET NC consists of two separate concrete units each one including a fan-shaped array of collimators. One unit views the plasma horizontally (10 LOS), and the other vertically (9 LOS). In each unit, the collimation can be remotely set up by the use of two pairs of rotatable steel cylinders with a choice of two aperture sizes $(21 \mathrm{~mm}$ and $10 \mathrm{~mm} \varnothing$ respectively). Each LOS is equipped with a set of three different detectors:

- NE213 liquid scintillator $(2.5 \mathrm{~mm} \varnothing, 10 \mathrm{~mm}$ thick) for the simultaneous measurement of $2.5 \mathrm{MeV}$ and $14 \mathrm{MeV}$ neutrons as well as gamma rays (pulse shape discrimination capability).

- $\mathrm{CsI}(\mathrm{Tl})$ photodiodes for measurements Hard $\mathrm{X}$ rays in the energy range $0.2-6 \mathrm{MeV}$.

- Bicron BC418 plastic scintillator $(2.5 \mathrm{~mm} \varnothing, 10 \mathrm{~mm}$ thick) for $14 \mathrm{MeV}$ neutron detection only with very low sensitivity to gamma radiation. The BC418 scintillators still work with an old analog acquisition electronics having several limitations:

-No raw data (i.e. scintillator pulses) storage for monitoring and reprocessing purposes.

-No provision of accurate pulse height spectra (PHS) during plasma discharges (analog discriminator modules provide counts in 4 energy bands only).

-Complex calibration procedure.

The NE213 detectors are coupled to an FPGA (Field Programmable Gated Array)-based digital acquisition system (14 bit ADC, $200 \mathrm{MS} / \mathrm{s}$ sampling rate) recently developed by ENEA. The digital system provides for all the missing features listed above, but the off-line processing is limited to $\sim 9 \times 10^{5} \mathrm{cps}$ (lab. tests) and the real time processing to $\sim 3 \times 10^{6} \mathrm{cps}$; DT count rates above such limits are expected in future DT campaigns. Moreover only a rough management of piled up events is implemented: pile-ups are recognized and their number used to calculate a correction factor that is applied to single neutron and gamma count rates. No attempt is made to recover the single pulses composing a pile-up event, to perform neutron gamma discrimination and to calculate their energy.

To address the insufficiencies of this diagnostic, an enhancement project was launched with two main objectives:

- Increase of the performances and reliability of the $14 \mathrm{MeV}$ neutron measurements performed by BC418 detectors.

- Assess the possibility of increasing the counting rate capabilities of the NC detection system based on NE213 detectors.

The first objective will be achieved by procuring, setting up, installing at JET and calibrating a new FPGA-based digital acquisition system provided with specific software tools for the treatment of piled up pulses. The new units will be put in parallel to the BC418 analog acquisition chain and will:

- Sustain count rates in the Mcps range and provide real time outputs

- Allow to store raw data for off -line reprocessing

- Provide an integrated and more reliable environment for detector monitoring and calibration

- Allow a better handling of piled-up events

The proposed upgrade will include:

- Digital acquisition for each BC418 detector;

- On-line data processing on FPGA (data reduction, pile-up processing, real time count rates depending on hardware architecture);

- Raw data mass storage on a local computers located in the NC cubicles area

- Off-line data processing (pile up processing, DT neutron count rates, pulse height spectrum, calibration, 
etc.) with dedicated software package running local computers.

The second objective will be supported by experimental tests at high DT neutron count rate, carried out for example at the Frascati Neutron Generator (FNG), with the new data acquisition units coupled to a spare detector unit including a BC418 scintillator and a NE213 scintillator.

If the increase in the NE213 maximum achievable count rate identified will be considered as a sufficient driver to carry out a further electronics upgrade for this detection system, new DAS units for the NC NE213 detectors will be procured, set-up, installed at JET and calibrated.

A simulation activity is also foreseen with the aim of setting up and benchmarking a Fokker-Plank simulation code to be used as predictive tool for neutron emissivity studies in future JET DT campaigns and to provide estimates of the neutron fluxes and spectra expected at the $\mathrm{NC}$ detector positions.

\section{Diagnostic upgrades for alpha particles}

\subsection{Gamma ray spectroscopy}

The $\alpha$-particles produced by the nuclear fusion reactions between deuterons and tritons are expected to provide the power for self-sustained DT-plasma burn by transferring their energy to the thermal plasma during their slowing down. Therefore the adequate confinement of $\alpha$-particles will be essential to obtain efficient heating of the bulk plasma and steady-state burning of reactor plasma. Consequently, the investigation of $\alpha$-particles behaviour for deciphering the main mechanisms of their slowing down, redistribution and losses, is a priority task for the planned deuterium- tritium experiments on JET in order to develop optimal plasma scenarios.

On JET the $\alpha$-particle diagnostic is based on the nuclear reaction ${ }^{9} \mathrm{Be}(\alpha, \mathrm{n} \gamma){ }^{12} \mathrm{C}$ between confined $\alpha$ particles and beryllium impurity ions typically present in the plasma. The applicability of gamma-ray diagnostics is strongly dependent on the fulfilment of rather strict requirements for the definition and characterization of the neutron and gamma radiation fields (detector Field-of-View, radiation shielding and attenuation, parasitic gamma-ray sources). For gamma ray spectrometry at the high DT neutron fluxes expected in the future high-power DT campaign on JET, specific improvements are needed in order to provide good quality measurements in the D-T campaign, characterized by a more challenging radiation environment.

In order to enable the gamma-ray spectroscopy diagnostic for $\alpha$-particle diagnostic during the DT campaigns the following goals should be achieved:

- Maximization of the signal-to-background ratio at the spectrometer detector.

- Establishing high count rate signal processing and energy-resolved gamma-ray detection.

These objectives can be accomplished by:

- Design, manufacturing and installation of a suitable assembly for the definition and control of the radiation fields (both neutron and gamma-ray) along the full line-of-sight of the gamma-ray spectrometer.

For proper gamma-ray measurements, the necessary reduction of the neutron flux of $14 \mathrm{MeV}$ neutrons reaching the detectors will be achieved by the manufacturing and installation of a set of $\mathrm{LiH}$ neutron attenuators. The choice of $\mathrm{LiH}$ material has the advantage of avoiding carbon-containing materials which lead to the production of inelastic scattering neutrons with energies $\mathrm{E}>5 \mathrm{MeV}$ from ${ }^{12} \mathrm{C}\left(\mathrm{n}, \mathrm{n}^{\prime} \gamma\right){ }^{12} \mathrm{C}$ reactions and, consequently, to an unwelcome background of $4.44 \mathrm{MeV} \gamma$-rays. LiH with a natural Li composition is compact, effective and well transparent to $\mathrm{MeV} \gamma$-rays. It does not produce interfering $\gamma$-rays in the high-energy range. The $\mathrm{LiH}$ attenuator should provide a reduction of the neutron flux by a factor of $10^{4}$ for $2.5 \mathrm{MeV}$ neutrons and by a factor of $10^{2}$ for $14 \mathrm{MeV}$ neutrons, respectively.

As in the DT experiments the gamma-ray detector must fulfil requirements for high count rate measurements, the existent BGO-detector should be replaced with new detector modules: detector module 1 (DM1) based on $\mathrm{LaBr}_{3}$ scintillator and detector module 2 (DM2) based on $\mathrm{CeBr}_{3}$ scintillator) and an associated digital data acquisition system. The new scintillators are characterized by short decay times $(\sim 20 \mathrm{~ns})$ and a high photons yields. The coupling of the scintillators with photomultiplier tubes in specially designed detector modules will permit the operation at count rates over $2 \mathrm{MHz}$. The high rate capability will be enabled by a dedicated pulse digitization system with a nominal 14-bit resolution.

The first option $\left(\mathrm{LaBr}_{3}\right)$ represents a reliable solution already manufactured and tested for JET gammaray spectrometry. The lanthanum bromide scintillation material has a good energy resolution, high sensitivity, short decay time and room temperature operability what makes it is a very good candidate for high resolution $\gamma$-ray diagnostics of JET plasmas. The main drawback of the $\mathrm{LaBr}_{3}$ scintillator is its use in low noise measurements due to its high intrinsic background noise. The background is caused by the $0.09 \%$ 
natural abundance of radioactive ${ }^{138} \mathrm{La}$ isotope with half-life $\sim 10^{11}$ years. The radioactive decay of the

${ }^{138} \mathrm{La}$ leads to $\gamma$-ray emission at $\sim 1.4 \mathrm{MeV}$ and $\sim 0.8 \mathrm{MeV}$ energies. Absorption of these gammas causes an internal activity of $\mathrm{LaBr}_{3}$ up to $\sim 10^{-3} \mathrm{~Bq} / \mathrm{cm}^{3} / \mathrm{keV}$. Therefore, besides ensuring an already tested component, the project attempts to provide also enhanced performance by including also a detector based on $\mathrm{CeBr}_{3}$ scintillator. This scintillator was found to fulfill low noise measurement conditions. It shows 30 times reduction in internal activity in comparison with $\mathrm{LaBr}_{3}$. The $\mathrm{CeBr}_{3}$ scintillator has a similar energy resolution, sensitivity and decay time as the $\mathrm{LaBr}_{3}$ scintillator. Moreover, the $\mathrm{CeBr}_{3}$ scintillator seems to be more resistant for gamma radiation than $\mathrm{LaBr}_{3}$. A $1 \mathrm{kGy}$ dose of gamma radiation deteriorates the yield of $\mathrm{LaBr}_{3}$ by $\sim 10 \%$ and worsens its energy resolution from 3.0 to $3.8 \%$, while is almost negligible for $\mathrm{CeBr}_{3}$. $\mathrm{CeBr}_{3}$ may also be more resistant for neutron radiation than $\mathrm{LaBr}_{3}$ because of the much lower neutron capture cross section in Ce than in La (at low neutron energy range, En<10keV).

The development of shielding, collimator and detector modules should be assisted by neutron-photon calculations which will provide radiation field assessment mainly at the detector position and detector response function. The envisaged modelling of DT alphas will be based on the Fokker-Planck code FIDIT (3D in constant-of-motion (COM) space) incorporating fast ion transport induced by Coulomb collisions in JET-like tokamaks with weak toroidal field ripples. The up-to-date version of FIDIT is suitable for simulating the time-dependent distributions of fast ions in realistic plasma equilibria and plasma parameters provided by the JET database. Previously, the code FIDIT was used for predictive modelling of confined and lost alpha particles in JET scenarios with monotonic and current hole equilibria. FIDIT calculations of the distribution of beam tritons gave a satisfactory explanation of the current hole effect on the spatial profiles of neutron emission in JET TTE experiments and served as a validation of the code. The simulation of the time evolution of gamma emission generated in nuclear reactions of impurity ions with alphas having energies above $1.7 \mathrm{MeV}$ in JET TTE was based on the numerical solution of a simplified 3D COM Fokker-Planck equation neglecting the diffusive transport. Both the present as well as a new version of the code FIDIT, which will include collisional diffusion effects, are proposed to be used for modelling the time-dependent distributions of beam deuterons produced during D blips in tritium plasmas, as well as of the DT fusion alphas in the envisaged DT campaigns.

By combining the alpha particle evolution codes with calculations of the plasma gamma-ray emission and detector response a procedure for generating synthetic gamma-ray spectra will be developed. This will allow for the validation of the numerical codes by comparing the synthetic and experimental gammaray spectra.

\subsection{Gamma ray Tomography}

In an ignited DT burning plasma the heating will be provided entirely by the slowing down of the 3.5 $\mathrm{MeV}$ fusion alpha particles, the confinement and control of which will be crucial. Besides the alpha particles, which will be the dominant suprathermal species on the next step tokamak ITER, there will be also other minority energetic ions produced by the auxiliary heating systems. These non-fusion born suprathermal particles must also be diagnosed and studied, as their confinement affects the plasma performance, as well as to avoid the serious damage they might cause to the tokamak first wall if lost from the plasma. All of the above has motivated the efforts to investigate the physical processes pertaining to fast ions in today's fusion experiments on D plasmas, with the final aim to understand and, eventually, control the physics governing the $\alpha$ particle behaviour in a burning DT plasma.

From a diagnostic point of view, high power DT plasma represents a rather harsh environment. For this reason, nuclear physics based diagnostics play a key role and interesting candidates are represented by high resolution Neutron Emission Spectroscopy (NES) and Gamma Ray emission spectroscopy (GRS). Neutrons of mean energy around $14 \mathrm{MeV}$ are produced by the DT reaction itself while gamma rays are emitted by reactions among alpha particles or other fast ions and light impurities, typically beryllium or carbon which are present in the plasma.

For operating the Gamma Ray Camera diagnostic at the high DT neutron fluxes expected in the next high-power DT campaign on JET and to improve its spectroscopic capability specific hardware improvements are planned to be put in place. In particular it is planned to:

- Enhance the existing spectroscopic and count rate capability by replacing the 19 CsI detector with new faster and better energy resolution detector modules. This is a challenging upgrade given the existing constraints in terms of available space for detectors and shielding. A possible solution is to the previously mentioned $\mathrm{LaBr}_{3}$ scintillators which are characterized by short decay times ( $\left.20 \mathrm{~ns}\right)$ and a high photon 
yield ( $\sim 60000$ photons/MeV) coupled to solid state photon detectors. The new detector will be able to sustain count rate in excess of $500 \mathrm{kHz}$ with an energy resolution equal or better than $5 \%$ at $1.1 \mathrm{MeV}$

- Implement a Control and Monitoring System (C\&M) of the detectors. This will be done if compatible with the limited available space.

- Refurbishment of the detector mechanics (housing, support, slider).

- Adapt and if necessary modify the existing digital data acquisition developed in a previous project, in order to sustain the high count rate measurements provided by the new fast detectors. Target values for the data acquisition are sampling frequency of $400 / 500 \mathrm{MHz}$ at 14 bit resolution.

\subsection{Diagnostic for the lost alphas}

The scintillator probe for lost ions is already now one of the main fast ion diagnostics at JET, and during future DT campaign will be a key systems to gain a better understanding of alpha particle physics. The diagnostic works on the magnetic spectrometer principle, where fast ions near the plasma boundary entering the probe through a collimator will hit different regions of a 2-D scintillator plate depending on their energy and the ratio of parallel and perpendicular velocity components, relative to the magnetic field. The diagnostic has been recently equipped with a fast scintillator material and a fast framing camera (Photron APX-i2) to record images with high space resolution. These last upgrades have shown good performance during the last campaign with moderate fluxes of escaping ions. The diagnostic is also equipped with a PMT with coarser spatial resolution but potentially higher temporal resolution, whose limited sampling rate $(5 \mathrm{kHz})$ prevents however the identification of fast MHD fluctuations in the escaping ion signals. As part of an ongoing upgrade, the PMT will be connected via a custom two-stage transimpedance amplifier to new digitisers with $2 \mathrm{MHz}$ bandwidth.

For an absolute calibration of the diagnostic to alphas, the scintillator screen will be characterized at operational temperature at the Spanish National Centre of Accelerators, and the transmittance of the light relay system from the torus to the diagnostic hall determined with an Ulbricht sphere positioned in-vessel.

These efforts will be supplemented with the development of a synthetic diagnostic, whose aim is to predict for given plasma parameters and heating type (ICRF, neutral beam injection, alphas) the expected signal seen by the probe. The synthetic diagnostic will include shadowing effect from nearby vessel wall components through a $3 \mathrm{D}$ model of the vessel wall.

\section{Instabilities Diagnostic Upgrades}

\subsection{Toroidal Alfven Mode system}

Instabilities in the Alfvén frequency range can be driven by fast ions (including fusion generated alpha particles) and can lead to their spatial redistribution and eventually fast radial transport that can affect the fusion performances and could damage the first wall of future fusion reactors. The understanding of the mechanisms of the mode stability is therefore of paramount importance for ITER and can also be used to control the alpha particle population itself.

At JET, the study of such modes, especially the Toroidal Alfvén Eigenmodes (TAEs), has been for the last two decades of high interest. AEs can be excited by means of in-vessel exciters (or antennas) and fast ions can be produced by additional heating like ICRH or NBI injections. A unique, state of the art, detection system allows in real-time the detection of TAEs of specific toroidal mode number(s) in the range $\mathrm{n}=0-15$, the measurement of their damping rate and amplitude and their tracking. The discrimination in real-time between the different modes of the frequency degenerate TAE spectrum is based on the successful implementation of an algorithm based on Sparse Representation of signals.

The system excites MHD modes around the TAE frequencies by performing a frequency sweep around the TAE frequency, which is calculated in Real Time by the AE Local Manager (AELM). The plasma response during frequency sweeps is extracted from the noise via synchronous detection of a series of magnetic coils and other relevant plasma quantities. The synchronous detection is performed analogically using electronic modules. The Real Time analysis of the synchronous signals through the AELM allows controlling the function generator in order to sweep the frequency around the AE frequency. When a resonance is measured the AELM locks onto it and sweeps the function generator frequency around that resonance. The implementation of the SparSpec analysis method into the AELM [6] allows one to track on pre-selected toroidal mode(s) $(n=+/-1-25)$ so avoiding to always track on the dominant modes. It is 
therefore technically possible, and it has been successfully demonstrated in the past campaigns, to measures more that 100 resonances of different $n$ numbers in a single JET discharge.

The actual system comprises 8 antennas asymmetrically located in the toroidal location. The 8 antennas were so far driven by a single $5 \mathrm{~kW}$ broadband (20-500 kHz) BONN-type amplifier (700V, 15A (peak)), which will be replaced with a new generation of amplifiers: D band power switching amplifiers of $4 \mathrm{~kW}$ each that can tolerate high reflected power. This will allow a more reliable operation and will provide the diagnostic with the potential to further increase the antenna current, hence TAE modes excitation. A new impedance matching system will be designed, procured and installed to optimize the antenna current and hence the coupling with the plasma over the whole frequency bandwidth. The minimum frequency bandwidth is $20-500 \mathrm{kHz}$ with the aim of extending to $10 \mathrm{kHz}-1 \mathrm{MHz}$ depending of the outcome of the amplifier development prototype phase.

\subsection{Reflectometer Upgrades}

The effects of the different isotopic composition on the plasma turbulence and related effects (such as the modifications in the ELM behaviour) will be one of the main topics of the D-T campaigns. Reflectometry measurements in the core region are paramount to better understand the mechanisms driving the turbulence and its associated transport in JET advanced scenarios. An ongoing upgrade project foresees adding an additional frequency band to the correlation reflectometer to allow the measurement of density fluctuations inner in the plasma, i.e. in the core region and even up to the high field side region in the most favourable cases. This is of prime interest to study various regimes of plasma turbulence, such as TEM (Trapped Electron Modes) and ITG (Ion Turbulence Gradient) regimes, and in particular assess their ballooning properties (i.e. asymmetry between the low field side and high field side regions).

The implementation will use a $12-18 \mathrm{GHz}$ source with a $\mathrm{x} 8$ multiplier, which means a probing frequency range of 96-144 GHz. The local oscillator and the plasma probing signals are generated by separated frequency synthesizers resulting in a single pure spectral lines providing the following main advantages: no spurious signals, no extraneous frequencies and therefore no intrinsic crosstalk on the received signal.

In addition, the radial electric field $\left(\boldsymbol{E}_{\boldsymbol{r}}\right)$ plays an important role in critical areas of tokamak science such as: L-H transition physics \& H-mode pedestal structure, turbulence suppression through equilibrium $\boldsymbol{E} \times \boldsymbol{B}$ shear and understanding of core rotation, momentum transport, and intrinsic rotation. Fast $\boldsymbol{E}_{\boldsymbol{r}}$ measurements also enable characterization of zonal flows and geodesic acoustic modes (GAMs). During past campaigns and in certain vertical target plasma shapes, Doppler backscattering (DBS) measurements were already possible, but a small modification of the microwave access front-end will enable $\boldsymbol{E}_{\boldsymbol{r}}$ measurements in future campaigns on a routine basis.

\section{Conclusions}

In the last decades the amount of data acquired by JET diagnostics has followed the Moore law, doubling about every two years. The record of data acquired per shot now exceeds 55 Gigabytes and the total warehouse is larger than 350 Terabytes. With the new upgrades and technological projects, the diagnostic capability is expected to keep improving and to adequately support the scientific programme of the next DT campaign, DTE2.

\section{Acknowledgement}

This work has been carried out within the framework of the EUROfusion Consortium and has received funding from the Euratom research and training programme 2014-2018 under grant agreement No 633053. The views and opinions expressed herein do not necessarily reflect those of the European Commission.

\section{References}

[1] A. Murari et al 2005 Plasma Phys. Control. Fusion 47 B249 doi:10.1088/0741-3335/47/12B/S19

[2] R. Felton et al Real-time measurement and control at JET experiment control

[3] V. Kiptily et al AIP Conference Proceedings 1612, 87 (2014); doi: 10.1063/1.4894029 


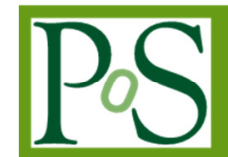

PROCEEDINGS

OF SCIENCE

[4] Lioure et al Fusion Engineering and Design 74 (2005) 141-146.

[5] B. Esposito et al Rev. Sci. Instrum. 75, 3550 (2004); http://dx.doi.org/10.1063/1.1785278

[6] D. Testa et al. EPL 92 (2010) 50001 